\title{
A common TLR1 polymorphism is associated with higher parasitaemia in a Southeast Asian population with Plasmodium falciparum malaria
}

William O. Hahn ${ }^{1 *}$ (D, Susanna Harju-Baker ${ }^{2}$, Laura K. Erdmann ${ }^{3,4}$, Srivicha Krudsood ${ }^{5}$, Kevin C. Kain 3,4, Mark M. Wurfel ${ }^{1+}$ and Wayne C. Liles ${ }^{1+}$

\begin{abstract}
Background: The factors leading to poor outcomes in malaria infection are incompletely understood. Common genetic variation exists in the human genes for Toll like receptors (TLRs) that alter host responses to pathogenassociated molecular patterns. Genetic variation in TLR1 and TLR6 could alter the risk of development of complicated malaria and ability of the host to control the parasite burden during acute Plasmodium falciparum infection.

Methods: Five single nucleotide polymorphisms in TLR1 and TLR6 in 432 patients with clinical P. falciparum monoinfection acquired on the Thai-Myanmar border were genotyped. Using logistic regression, associations with the development of complicated malaria and the percentage of infected erythrocytes (parasitaemia) on the day of presentation to clinical care (day zero) were tested.

Results: Genotypes carrying the T (major) allele of TLR1 rs5743551 - an allele associated with improved outcomes in sepsis - were associated with higher parasitaemia measured on day zero $(p=0.03)$.

Discussion: Since malaria exerts strong genetic pressure on the human genome, protection from parasitaemia associated with TLR1 rs5743551 may account for the maintenance of an allele associated with poor outcomes in Caucasians with sepsis.

Conclusion: These data suggest that genetic variation in TLR1 has effects on the host response to Plasmodium falciparum malaria in Asian populations. Genotypes from TLR6 showed no evidence of association with either complicated malaria or parasite burden.
\end{abstract}

Keywords: Plasmodium falciparum, Single nucleotide polymorphisms, Toll-like receptor 1, Toll-like receptor 6, Sepsis, Parasitaemia

\section{Background}

Malaria is estimated to cause approximately 850,000 deaths per year with most mortality occurring before the age of 5 years [1]. Since death occurs prior to the

\footnotetext{
*Correspondence: willhahn@uw.edu; willhahn@u.washington.edu ${ }^{\dagger}$ Mark M. Wurfel and Wayne C. Liles contributed equally to this study and manuscript

${ }^{1}$ Division of Allergy and Infectious Diseases, Department of Medicine, Harborview Medical Center, University of Washington, Box 359640, Seattle, WA, USA

Full list of author information is available at the end of the article
}

age of reproduction, the malaria parasite is thought to exert strong selective pressure on the human genome. The clearest example is the sickle cell trait where the heterozygote state provides protection against malaria whereas homozygosity for sickle cell trait (sickle cell disease) is uniformly fatal. The high prevalence of several independently arising alleles causing sickle cell trait in different populations implies a strong selective pressure on the human genome [2]. While the immunologic host determinants of susceptibility to malaria remain 
incompletely understood, family-based longitudinal studies have demonstrated that susceptibility to clinical malaria is partially heritable [3].

The role of innate immunity and, specifically, innate immune responses in host defence against malaria has been reviewed previously [4]. Common genetic variations in TLR genes have been associated with disease susceptibility in humans to a wide variety of pathogens [5]. Polymorphisms in innate sensing pathways have also been implicated in host susceptibility to malaria [6]. There are several components of the malaria parasite that have been implicated as pathogen-associated molecular patterns (PAMPs) recognizable by the innate immune system, including glycosylphosphatidylinositol (GPI) anchors on the surface membranes of infected red blood cells (RBCs) and DNA-haemozoin complexes within the cytoplasm [7, 8]. GPI initiated signals are thought occur via TLR1/2 or TLR2/TLR-6 heterodimers and DNAhemozoin complexes via TLR9 [8-10]. TLR1 is part of a complex of TLR receptors that includes TLR1, TLR6, and TLR10, and is thought to have arisen via a gene duplication event [11].

Since Plasmodium is a well-described cause of the sepsis syndrome, the possible contribution of TLR polymorphisms to clinical outcomes in malaria also has potential explanatory power $[12,13]$. In a cohort of patients with sepsis and septic shock from an area with no ongoing malaria transmission, it has been shown that common variation in the gene for TLR1, the G (minor) allele of $T L R 1_{7202 \mathrm{~A} / \mathrm{G}}$ (rs5743551) is associated with a large increase in the severity of organ dysfunction and an increased risk of death [14]. Others have observed similar biologic effects of this hyperfunctional variant and have shown this allele to be associated with increased risk for clinical tuberculosis, leprosy and frequency of leprosy reversal reactions $[15,16]$. The minor allele of $T L R 1_{7202 \mathrm{~A} / \mathrm{G}}$ has been also been associated with increased Interleukin-6 (IL-6) production and decreased regulatory $\mathrm{T}$ cell $(\mathrm{CD} 4+, \mathrm{CD} 25+)$ mediated suppression of proliferation utilizing in vitro culture systems [17]. The $T L R 1_{7202 \mathrm{~A} / \mathrm{G}}$ (minor allele) is a non-coding SNP that sits within the $5^{\prime}$ upstream region of the TLR1 gene and, thus, cannot have a direct effect on TLR1 protein function. It has been previously demonstrated, however, that the rs 5743551 SNP is associated with increased secretion of soluble mediators of inflammation in response to stimulation with Pam3CysSerLys4 (Pam3CSK4), a specific ligand for TLR1/TLR2 heterodimers [14]. One potential explanation for the observed hyper responsiveness is that TLR1 rs5743551 is in LD with several coding polymorphisms that strongly increase cell surface expression of TLR1.

These data suggest that polymorphisms in TLR1 have functional consequences in immunologic signalling and influence the host response to a broad range of microbial pathogens. Given the potential role of TLR1 in inflammatory responses to malaria, it was hypothesized that the presence of the minor $T L R 1_{A 7202 G}$ allele would be associated with malaria severity and the magnitude of parasitaemia.

\section{Methods}

\section{Malaria patients}

Patients (age $>13$ years) were enrolled from a population presenting with febrile illness to clinical sites for Hospital for Tropical Disease (Mahidol University) on the ThaiMyanmar border found to be infected with Plasmodium falciparum malaria. Study recruitment was from 20002004, a period in which malaria was endemic. Patients were excluded if they had another plausible medical explanation for fever (e.g., lobar pneumonia). The age of 13 was selected as subjects were originally recruited to participate in a previously reported clinical trial [18]. Both thick and thin smears were performed on whole blood specimens from all patients. Enrolment occurred on the basis of unblinded smear microscopy, but this was confirmed with PCR (as previously described) [19]. Patients with mixed infections were excluded. Patients were initially classified into uncomplicated malaria (UM), severe malaria or cerebral malaria on the basis of WHO criteria [20].

An expert microscopist blinded to other diagnostic testing confirmed the presence of $P$. falciparum peripheral blood smear after enrolment and performed quantitation of the parasite. Species level infection was also confirmed with PCR as previously described [21]. Parasite density was determined by counting the number of parasites per 200 white blood cells for thick blood films or per 1000 RBCs for thin blood films.

The institutional review board of the Faculty of Tropical Medicine, Mahidol University approved this study. Verbal and written explanations were provided in the patient's' native language, and informed consent was obtained from all patients or their legal guardians prior to specimen collection.

\section{Genotyping}

TLR1 (rs5743551, rs5743614, rs5743618) and TLR6 (rs3775073, rs5743808) in the malaria cases were genotyped using Taqman-based real-time polymerase chain reaction (RT-PCR) as previously described [22]. Primerprobe sets were obtained from the Applied Biosystems repository $\left(T L R 1_{-7202 A / G}\right)$ or synthesized $\left(T L R 1_{1517 \mathrm{G} / \mathrm{A}}\right.$, forward primer: AGCAGCCTTTCTGTATTGATCATTGA, reverse primer: CATCTTCTGGCAGCTCTGGAA, probe: CACCCATC[G/A]GCTGAT; $T L R 1_{1804 \mathrm{G} / \mathrm{T}}$, forward primer: GGTGTTGGCTGTGACTGTGA, reverse 
primer: GCACACCATCCTGAGATACCA, probe: CCT CTGCA[G/T]CTACTT) and run according to the recommended guidelines on an ABI Prism 7900HT (Applied Biosystems, Inc., Foster City, CA, USA). $2 \%$ of the samples were replicated for quality control.

\section{Statistical analysis}

The two phenotypes tested for association with TLR1 and TLR6 genotypes were:

(1) Complicated malaria disease versus uncomplicated disease, and (2) $\log _{10}$-transformed measurements of parasitaemia on day 0 for each subject. Observed genotype frequencies were compared with expected frequencies to test for deviations from Hardy-Weinberg equilibrium as previously described [23]. Inspection of the genotype frequencies by ethnicity revealed that patients that were self-declared "Karen" exhibited genotype frequencies highly divergent from the other major ethnic groups (data not shown). Patients of Karen ethnicity were excluded to avoid confounding due coexistent but unrelated population-specific differences in genotype frequencies and phenotype prevalence. Cambodian and Nepali patients were also excluded due to very low population sizes. To identify associations between TLR1 and TLR6 genotypes and the presence of complicated malaria in the Burmese, Mon, and Thai patients, logistic regression was used, assuming an additive effect as previous work demonstrated an intermediate functional phenotype in heterozygotes [14]. To test for an association between TLR 1 and TLR6 genotypes and day $0 \log _{10}$ transformed parasitaemia, linear regression was used assuming an additive effect. For associations detected in the un-adjusted analyses, multiple linear regression analyses were performed adjusting for age and gender. Finally, to assess whether genotypic effects were consistent across ethnic groups, sensitivity analyses were performed with stratification on the basis of each of the three major ethnic groups (Mon, Burmese, and Thai). Statistical significance was set at $\mathrm{p}<0.05$. Analyses were performed using Stata 10.0 (Stata Corp., College Station, TX, USA).

\section{Results}

TLR1 genotype frequency and susceptibility to complicated malaria

Common genetic variants within TLR1 and TLR6 are associated with altered innate immune responses to synthetic bacterial lipoproteins [14]. Functional genetic variation within TLR1 and TLR6 could therefore be associated with severity of malarial illness and/or the degree of parasitaemia observed in each patient on the day patients were diagnosed with clinical malaria. To test this hypothesis five different single nucleotide polymorphisms (SNPs) were genotyped, three in TLR1 and two in TLR6 (Fig. 1), in a group of 435 patients enrolled in northern Thailand with PCR-confirmed infection with P. falciparum.

Self-reported race/ethnicity in the studied group revealed the existence of several populations with the majority of patients declaring Thai ancestry $(41.8 \%)$ followed by Mon (29.0 \%), Karen (16.3\%), Burmese (12.2 \%), and, to a much lesser extent Cambodian and Nepali (Tables 1,2). The patients were young with a mean age of 27 years. In terms of severity of illness, $59 \%$ had UM while $19.8 \%$ had cerebral malaria and $21.2 \%$ had complicated disease. Mean parasitaemia on day 0 was nearly 80,000 parasites/uL and ranged from 13 to over $1 \times 10^{6}$ parasites/uL. As expected, disease severity and parasitaemia were correlated. Mean parasitaemia in patients with cerebral or complicated malaria was roughly an order of magnitude higher than in patients with uncomplicated malaria $(\mathrm{p}<0.01)$ (Table 2$)$. Given the relatively small number of patients with complicated disease and the assumption that TLR responses are likely to be an early event in malaria host defense within the causal pathway for both forms of complicated malaria, it was decided to pool cerebral and complicated malaria for the purposes of analysis. This approach is consistent with natural history studies in African children where severe clinical malaria exists as a series of overlapping syndromes rather than discrete clinical phenotypes [24].

Consistent with prior reports documenting this divergence using other genetic markers, genotype frequencies for patients of Karen ethnicity were markedly different from other major ethnic groups and are divergent from frequencies observed in reference Asian populations [25, 26 . This was not due to assay variability, as genotyping was successful with over $98 \%$ of all genotypes called with excellent agreement between replicates (>99 \%) and all genotype frequencies were found to be consistent with Hardy-Weinberg equilibrium. Given the likelihood that associations between TLR1 or TLR6 genotype frequencies and malaria phenotypes would be confounded by major underlying population differences in genetic

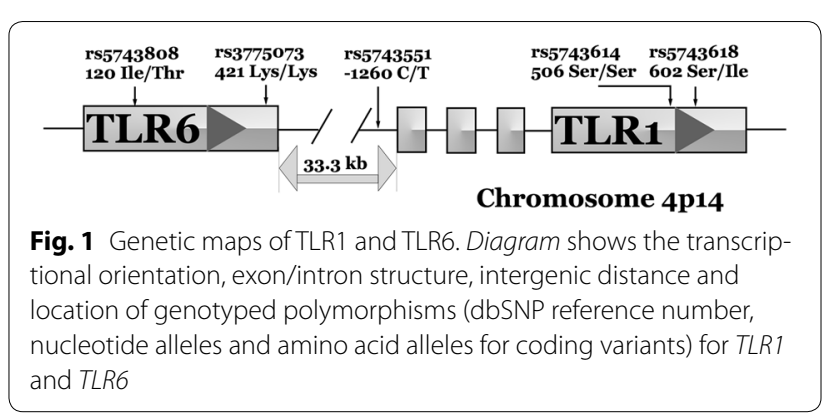


Table 1 Demographic characteristics of enrolled patients

\begin{tabular}{ll}
\hline $\mathrm{N}$ & 432 \\
Age, mean (std dev) & $27(11.1)$ \\
Ethnicity, $\mathrm{n}(\%)$ & \\
Thai & $182(41.8)$ \\
Mon & $126(29.0)$ \\
Karen & $71(16.3)$ \\
Burma & $53(12.2)$ \\
Cambodia & $2(0.5)$ \\
Nepali & $1(0.2)$ \\
\hline
\end{tabular}

Table 2 Clinical characteristics of enrolled patients

\begin{tabular}{lrllll}
\hline & N & Age & Sex & & $\begin{array}{l}\text { Do Parasitemia } \\
\text { (/ul) }\end{array}$ \\
\cline { 4 - 5 } & & $\begin{array}{l}\text { Median } \\
\text { (range) }\end{array}$ & $\% \mathbf{F}$ & $\% \mathbf{M}$ & Median (range) \\
\hline Uncomplicated & 257 & $25(2-65)$ & 32.3 & 67.7 & $7890(13-422,400)$ \\
Severe & 92 & $22(12-59)$ & 25.3 & 74.7 & $\begin{array}{c}71,400(350- \\
944,240)\end{array}$ \\
Cerebral & 86 & $26(14-61)$ & 19.4 & 80.6 & $\begin{array}{c}95,790(94- \\
1,188,720)\end{array}$ \\
\hline
\end{tabular}

architecture, analyses for the Karen subjects were performed separately from the other ethnic groups. Patients who self-reported Burmese, Mon, or Thai ethnicity were analysed as a group.

A trend towards an association between the "major" $\mathrm{T}$ allele of TLR1 rs5743551 (associated with less inflammatory responses in other systems) and susceptibility to complicated malaria [OR $(\mathrm{CI})=1.26(0.93-1.71)$, $\mathrm{p}=0.14$, Table 3] was observed. When analysed within each ethnic group, all three groups (Burma, Mon, and Thai) exhibited trends of similar direction and magnitude but none met pre-specified criteria for statistical significance (data not shown). No major trends were observed with any other polymorphism tested nor was there any significant association observed between any genotype and susceptibility to complicated malaria in subjects of Karen ethnicity.

\section{$T L R 1_{-7202 \mathrm{~A} / \mathrm{G}}$ is associated with elevated malaria parasitaemia}

Associations between malaria parasitaemia measured on day 0 of enrollment and TLR1 and TLR6 genotypes were also tested. Table 3 shows an association between increasing copy number of the T-allele (major allele) of TLR1 rs5743551 and higher levels of parasitaemia. A nearly two-fold increase in mean parasitaemia in subjects carrying the "major" TT genotype relative to those carrying the "minor" GG genotype (Table 3) was observed.
Notably, when analysed by ethnicity, all three major ethnic groups demonstrated a similar trend with the major allele with the strongest effect observed in subjects of Mon ethnicity ( $\mathrm{p}=0.03$ ) (Fig. 2). A similar trend was observed with TLR1 rs5743614 although this did not meet statistical significance. No other genotypes demonstrated an association with parasitaemia. In addition, no genotypes were found to associate with day 0 parasitaemia in subjects of Karen ethnicity.

\section{Discussion}

Early host innate immune inflammatory responses are likely to play an important role in determining the clinical course after infection with $P$. falciparum. It was determined that TLR1 polymorphisms previously demonstrated to be associated with functional changes in immunologic signaling were associated with different levels of host control of $P$. falciparum in patients with clinical malaria. In the studied patient population, the GG (minor) allele of rs5743551 was associated with a parasite density roughly half of that of patients with the TT (major) allele. The G (minor) allele has been associated with increased inflammatory cytokine production in response to bacterial lipopeptides that are recognized by TLR1/2 heterodimers [14]. The direction of this association with the $\mathrm{G}$ (minor) allele in the studied population of patients with clinical malaria, however, is opposite the direction in sepsis patients where the presence of the $\mathrm{T}$ (major) allele was associated with improved clinical outcomes. Furthermore, the density of the pathogen is different in patients with the $\mathrm{G}$ (minor) allele with respect to malaria and sepsis, as carriers of the minor $\mathrm{G}$ allele were more likely to have blood cultures with bacterial growth (a proxy for degree of bacterial burden) compared to carriers of the major $\mathrm{T}$ allele whereas the minor $\mathrm{G}$ allele was associated with fewer parasites per millilitre of blood. This implies that the influence of innate signaling molecules on the ability of the host to control an infectious process is more than simply the degree of inflammatory mediators produced. The $\mathrm{G}$ (minor) allele may have persisted in the population because early inflammation may be important to control parasite replication during acute malaria infection, whereas hyperresponsiveness to other PAMPS is detrimental in other disease states (e.g. bacterial sepsis).

Investigations into the role of TLR signalling in malaria infection using murine models have revealed conflicting results. Both TLR1 and TLR6 signal via forming heterodimers with TLR2 $[9,10]$ All TLRs except for TLR3 signal via the adaptor protein MyD88 and disruption in MyD88 and TLR2 disrupts signaling cascades generated by TLR1 and TLR6 [27]. During infection with Plasmodium berghei ANKA strain (a model for cerebral malaria), 
Table 3 Genotype associations

\begin{tabular}{|c|c|c|c|c|c|c|c|c|}
\hline \multirow[t]{2}{*}{ Gene } & \multirow[t]{2}{*}{ Genotype } & \multicolumn{4}{|l|}{ Malaria type } & \multicolumn{3}{|c|}{ Parasitemia (day 0 ) } \\
\hline & & Uncomplicated & Complicated & $\mathrm{OR}(\mathrm{Cl})$ & $p$ & Mean & $\beta$ & $p$ \\
\hline \multirow[t]{12}{*}{$T L R 1$} & rs5743551 & & & & & & & \\
\hline & $\mathrm{CC}$ & $59(64.8)$ & $32(35.2)$ & $1.26(0.93-1.71)$ & 0.14 & $57,224(91,846)$ & 0.16 & 0.042 \\
\hline & $\mathrm{CT}$ & $107(58.5)$ & $76(41.5)$ & & & $73,597(142,004)$ & & \\
\hline & $\mathrm{TT}$ & $44(53.7)$ & $38(46.3)$ & & & $106,744(190,805)$ & & \\
\hline & rs5743614 & & & & & & & \\
\hline & AA (Ser/Ser) & $59(62.8)$ & $35(37.2)$ & $1.17(0.86-1.59)$ & 0.32 & $57,116(89,419)$ & 0.14 & 0.078 \\
\hline & AG (Ser/Ser) & $110(58.5)$ & $78(41.5)$ & & & $73,087(142,004)$ & & \\
\hline & GG (Ser/Ser) & $41(55.4)$ & $33(44.6)$ & & & $111,539(198,734)$ & & \\
\hline & rs5743618 & & & & & & & \\
\hline & TT (lle/lle) & $202(58.6)$ & $143(41.5)$ & $1.41(0.48-4.12)$ & 0.53 & $79,114(147,719)$ & -0.03 & 0.91 \\
\hline & GT (Ser/lle) & $7(50.0)$ & $7(50.0)$ & & & $55,513(73,399)$ & & \\
\hline & GG (Ser/Ser) & 0 & 0 & & & - & & \\
\hline \multirow[t]{8}{*}{ TLR6 } & rs3775073 & & & & & & & \\
\hline & TT (Lys/Lys) & $55(57.9)$ & $40(42.1)$ & $0.89(0.66-1.18)$ & 0.42 & $87,461(143,708)$ & 0.07 & 0.35 \\
\hline & TC (Lys/Lys) & $106(63.1)$ & $62(36.9)$ & & & $81,825(167,173)$ & & \\
\hline & CC (Lys/Lys) & $49(52.1)$ & $45(47.9)$ & & & $61,273(99,746)$ & & \\
\hline & rs5743808 & & & & & & & \\
\hline & AA (lle/lle) & $158(58.5)$ & $112(41.5)$ & $0.96(0.62-1.48)$ & 0.85 & $79,744(150,930)$ & -0.04 & 0.74 \\
\hline & GA (Thr/lle) & $47(57.3)$ & $35(42.7)$ & & & $73,177(132,271)$ & & \\
\hline & GG (Thr/Thr) & $5(71.4)$ & $2(28.6)$ & & & $67,254(77,658)$ & & \\
\hline
\end{tabular}

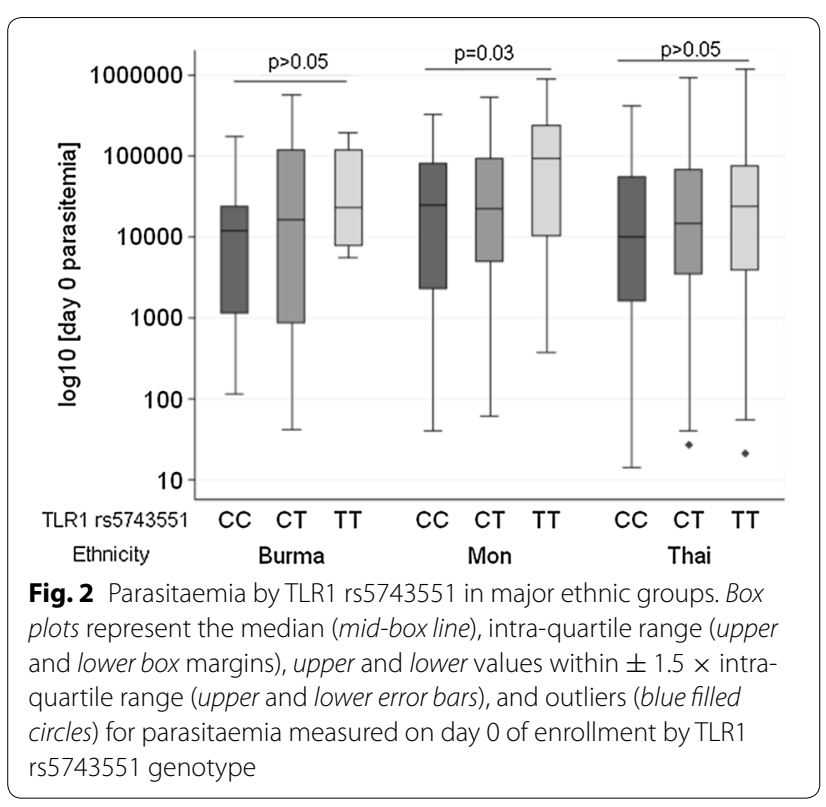

Tlr2-/ - and Myd88-/ - mice have been shown by some groups to be protected from development of cerebral malaria, although the effects of TLR2 have not been consistently observed and may depend on the genetic background of the mice [28-31]. Infection with Plasmodium yoelii, a model of acute uncomplicated malaria, had either a strong phenotype with impaired survival in Tlr2-/-, and Myd88-1- mice or no observable phenotypic difference [32, 33]. It is likely that there are multiple innate mechanisms that contribute to the host response against malaria infection, and single knockout inbred mice may not recapitulate the complexity of human disease.

In an effort to clarify the role of TLRs in human malaria, several studies have used genetic approaches attempting to detect associations between common genetic variants in the genes for TLRs and clinical outcomes in patients with malaria. In a group of malaria cases from northern Thailand with confirmed $P$. falciparum infection, none of the genetic variants tested were significantly associated with development of complicated malaria (Table 3), whereas increasing copy numbers of the major $\mathrm{T}$ allele of TLR1 rs5743551 demonstrated a trend towards higher odds of complicated disease $(\mathrm{p}=0.13)$. Notably, while this polymorphism is in high linkage disequilibrium (LD) with TLR1 rs5743551 in Caucasian subjects, it was determined that in the Southeast Asian subjects from Northern Thailand that TLR1 rs5743618 is in very weak LD with TLR1 rs5743551 and is found at very low frequency [14].

The finding that TLR1 rs5743618 is associated with higher parasitaemia on day zero in an Asian population is similar to an investigation by a Brazilian group that 
demonstrated an association between the $G$ allele of TLR1 rs5743618 and increased risk of developing symptomatic malaria after infection with Plasmodium [33]. The Brazilian group did not genotype TLR1 rs5743551 in their study, so direct comparisons between their study and the studied population cannot be made. However, taken together, these studies suggest that genetic variation in TLR1 affects host susceptibility to malaria disease severity, while variation in TLR6 is less likely to play a significant role in these processes. This association is further supported by a study in a population in Cameroon that reported a different SNP in TLR1 (rs4833095) was also associated with increased parasite burden, whereas a SNP in TLR6 was not associated with any clinical phenotype [34].

Stronger evidence that TLR1 rs5743551 may influence host responses to malaria is provided by the finding of a significant association between the copy number of the $\mathrm{T}$ allele of this polymorphism and increased parasitaemia measured on day 0 of enrollment. Subjects carrying the TT genotype had a nearly two-fold higher parasitaemia than subjects carrying the CC genotype (Table 3 ). Segregated analysis by ethnicity showed that the strongest effect of the $\mathrm{T}$ (minor) allele was present in the subjects of Mon ethnicity. This finding differs from those of the Brazilian group who found no association between TLR1 variation and parasitaemia. In contrast, the population in Cameroon demonstrated a similar association with TLR1 polymorphism associated with differences in parasite load. Explanations for the differing findings may be due to the different TLR1 variants tested but could also include marked differences in environment (Brazil versus Thailand), and underlying population genomic differences. Furthermore, in the Brazilian study a substantial minority of patients were infected with P. vivax (39\%), whereas the population in Cameroon was similar to the studied population in that it was a population uniformly infected with P. falciparum. Several groups have demonstrated different host response to the various Plasmodium species. For example, higher levels of IL-10 have been documented in humans subjects infected with Plasmodium vivax when compared to individuals infected with P. falciparum [31-33].

\section{Conclusions}

The particular strengths of the present study include detailed clinical information on a population uniformly infected with PCR confirmed P. falciparum. The current study is the first to document an association of TLR1 rs5743551 with a clinical outcome in an Asian population with malaria. TLR6 has never been strongly implicated in the host response to malaria and no association with TLR6 SNPs and clinical outcome in malaria were found.

These data demonstrate that the $\mathrm{G}$ allele of rs5743551 in TLR1 is associated with the host control of parasitaemia in Asian patients with acute febrile malaria. This assertion is consistent with the demonstrated ability of malarial GPI to activate cells through TLR2/TLR1 heterodimers [4]. From an evolutionary standpoint, this suggests that G allele of TLR1 rs5743551 may persist in higher frequencies in non-Caucasian populations because of a beneficial effect on host fitness in environments with high prevalence of malaria. This is the first plausible explanation for the relatively high frequency of an allele that is associated with worse outcomes in tuberculosis, sepsis, and leprosy [14-16]. While parasitaemia is generally correlated with complicated disease, high parasitaemia alone is neither necessary nor sufficient to account for poor patient outcomes and other host factors likely influence clinical outcomes [24]. These findings stress the complex interplay between the human genome and endemic pathogens.

\section{Authors' contributions}

WOH, LKE, KCK, MWW, and WCL planned and designed the study. WOH, MWW, and WCL created the first draft of the manuscript. MWW and SHB performed the SNP genotyping. MWW performed the initial statistical analysis. SK helped to enroll the cohort included in the study. All authors read and approved the final manuscript.

\section{Author details}

1 Division of Allergy and Infectious Diseases, Department of Medicine, Harborview Medical Center, University of Washington, Box 359640, Seattle, WA, USA. ${ }^{2}$ Division of Pulmonary Critical Care Medicine, Department of Medicine, Harborview Medical Center, University of Washington, Seattle, WA, USA. ${ }^{3}$ Tropical Disease Unit, Department of Medicine, University Health NetworkToronto General Hospital, Toronto, Ontario, Canada. ${ }^{4}$ SAR Laboratories, Sandra Rotman Centre for Global Health, University of Toronto, Toronto, Ontario, Canada. ${ }^{5}$ Clinical Malaria Research Unit, Faculty of Tropical Medicine, Mahidol University, Bangkok, Thailand.

\section{Acknowledgements}

The authors would like to acknowledge Professor Sornchai Looareesuwan University of Mahidol; deceased) who was instrumental in the development of the study cohort for this manuscript. This study was funded in part by the Canadian Institutes of Health Research MOP-13721, MOP-115160 and MOP136813 (KCK), CIHR MD/PhD Studentship (LE), and the CIHR Canada Research Chairs program (KCK, WCL).

\section{Competing interests}

The authors declare that they have no competing interests.

Received: 16 October 2015 Accepted: 22 December 2015

Published online: 06 January 2016

\section{References}

1. Murray CJL, Ortblad KF, Guinovart C, Lim SS, Wolock TM, Roberts DA, et al. Global, regional, and national incidence and mortality for HIV, tuberculosis, and malaria during 1990-2013: a systematic analysis for the global burden of disease study 2013. Lancet. 2014;384:1005-70.

2. Kwiatkowski DP. How malaria has affected the human genome and what human genetics can teach us about malaria. Am J Hum Genet. 2005;77:171-92 
3. Phimpraphi W, Paul R, Witoonpanich B, Turbpaiboon C, Peerapittayamongkol C, Louicharoen C, et al. Heritability of P. falciparum and $P$. vivax malaria in a Karen population in Thailand. PLoS One. 2008;3:e3887.

4. Gazzinelli RT, Kalantari P, Fitzgerald KA, Golenbock DT. Innate sensing of malaria parasites. Nat Rev Immunol. 2014;14:744-57.

5. Skevaki C, Pararas M, Kostelidou K, Tsakris A, Routsias JG. Single nucleotide polymorphisms of Toll-like receptors and susceptibility to infectious diseases. Clin Exp Immunol. 2015;180:165-77.

6. Corr SC, O'Neill LAJ. Genetic variation in Toll-like receptor signalling and the risk of inflammatory and immune diseases. J Innate Immun. 2009;1:350-7.

7. Sharma S, DeOliveira RB, Kalantari P, Parroche P, Goutagny N, Jiang Z, et al. Innate immune recognition of an AT-rich stem-loop DNA motif in the Plasmodium falciparum genome. Immunity. 2011;35:194-207.

8. Schofield L, Novakovic S, Gerold P, Schwarz RT, McConville MJ, Tachado SD. Glycosylphosphatidylinositol toxin of Plasmodium up-regulates intercellular adhesion molecule-1, vascular cell adhesion molecule-1, and E-selectin expression in vascular endothelial cells and increases leukocyte and parasite cytoadherence via tyrosine kinase-dependent signal transduction. J Immunol. 1996;156:1886-96.

9. Krishnegowda G, Hajjar AM, Zhu J, Douglass EJ, Uematsu S, Akira S, et al. Induction of proinflammatory responses in macrophages by the glycosylphosphatidylinositols of Plasmodium falciparum: cell signaling receptors, glycosylphosphatidylinositol (GPI) structural requirement, and regulation of GPI activity. J Biol Chem. 2005;280:8606-16.

10. Zhu J, Krishnegowda G, Li G, Gowda DC. Proinflammatory responses by glycosylphosphatidylinositols (GPIs) of Plasmodium falciparum are mainly mediated through the recognition of TLR2/TLR1. Exp Parasitol. 2011;128:205-11.

11. Georgel P, Macquin C, Bahram S. The heterogeneous allelic repertoire of human toll-like receptor (TLR) genes. PLoS One. 2009;4:e7803.

12. Jacob ST, Banura P, Baeten JM, Moore CC, Meya D, Nakiyingi L, et al. The impact of early monitored management on survival in hospitalized adult Ugandan patients with severe sepsis: a prospective intervention study. Crit Care Med. 2012:40:2050-8.

13. Bruneel F, Hocqueloux L, Alberti C, Wolff M, Chevret S, Bédos J-P, et al. The clinical spectrum of severe imported falciparum malaria in the intensive care unit: report of 188 cases in adults. Am J Respir Crit Care Med. 2003; 167:684-9.

14. Wurfel MM, Gordon AC, Holden TD, Radella F, Strout J, Kajikawa O, et al. Toll-like receptor 1 polymorphisms affect innate immune responses and outcomes in sepsis. Am J Respir Crit Care Med. 2008;178:710-20.

15. Hawn TR, Misch EA, Dunstan SJ, Thwaites GE, Lan NT, Quy HT, et al. A common human TLR1 polymorphism regulates the innate immune response to lipopeptides. Eur J Immunol. 2007;37:2280-9.

16. Misch EA, Macdonald M, Ranjit C, Sapkota BR, Wells RD, Siddiqui MR, et al. Human TLR1 deficiency is associated with impaired mycobacterial signaling and protection from leprosy reversal reaction. PLoS Negl Trop Dis. 2008;2:e231.

17. Mikacenic C, Schneider A, Radella F, Buckner JH, Wurfel MM. Cutting edge: genetic variation in TLR1 is associated with Pam3CSK4-induced effector $T$ cell resistance to regulatory $T$ cell suppression. J Immunol. 2014;193:5786-90.

18. Ashley EA, McGready R, Hutagalung R, Phaiphun L, Slight T, Proux S, et al. A randomized, controlled study of a simple, once-daily regimen of dihydroartemisinin-piperaquine for the treatment of uncomplicated, multidrug-resistant falciparum malaria. Clin Infect Dis. 2005;41:425-32.

19. Conroy AL, Lafferty El, Lovegrove FE, Krudsood S, Tangpukdee N, Liles WC, Kain KC. Whole blood angiopoietin-1 and -2 levels discriminate cerebral and severe (non-cerebral) malaria from uncomplicated malaria. Malar J. 2009;8:295.

20. World Health Organization. Communicable diseases cluster. Severe falciparum malaria. Trans R Soc Trop Med Hyg. 2000;94(Suppl 1):S1-90.

21. Perandin F, Manca N, Calderaro A, Piccolo G, Galati L, Ricci L, et al. Development of a real-time PCR assay for detection of Plasmodium falciparum, Plasmodium vivax, and Plasmodium ovale for routine clinical diagnosis. J Clin Microbiol. 2004;42:1214-9.

22. Livak KJ. Allelic discrimination using fluorogenic probes and the $5^{\prime}$ nuclease assay. Genet Anal. 1999;14:143-9.

23. Guo SW, Thompson EA. Performing the exact test of Hardy-Weinberg proportion for multiple alleles. Biometrics. 1992;48:361-72.

24. Gonçalves BP, Huang CY, Morrison R, Holte S, Kabyemela E, Prevots DR, et al. Parasite burden and severity of malaria in Tanzanian children. N Engl J Med. 2014;370:1799-808.

25. Besaggio D, Fuselli S, Srikummool M, Kampuansai J, Castrì L, Tyler-Smith C, Seielstad M, et al. Genetic variation in Northern Thailand Hill Tribes: origins and relationships with social structure and linguistic differences. BMC Evol Biol. 2007;7(Suppl 2):S12.

26. Summerer $M$, Horst J, Erhart $G$, Weißensteiner $H$, Schönherr $S$, Pacher $D$, et al. Large-scale mitochondrial DNA analysis in Southeast Asia reveals evolutionary effects of cultural isolation in the multi-ethnic population of Myanmar. BMC Evol Biol. 2014;14:17.

27. Takeda K, Akira S. Toll-like receptors. Curr Protoc Immunol. 2015;109:1-10.

28. Togbe D, Schofield L, Grau GE, Schnyder B, Boissay V, Charron S, et al. Murine cerebral malaria development is independent of toll-like receptor signaling. Am J Pathol. 2007;170:1640-8.

29. Griffith JW, O'Connor C, Bernard K, Town T, Goldstein DR, Bucala R. Toll-like receptor modulation of murine cerebral malaria is dependent on the genetic background of the host. J Infect Dis. 2007;196:1553-64.

30. Lepenies B, Cramer JP, Burchard GD, Wagner H, Kirschning CJ, Jacobs T. Induction of experimental cerebral malaria is independent of TLR2/4/9. Med Microbiol Immunol. 2008;197:39-44.

31. Geurts N, Martens E, Verhenne S, Lays N, Thijs G, Magez S, et al. Insufficiently defined genetic background confounds phenotypes in transgenic studies as exemplified by malaria infection in TIr9 knockout mice. PLoS One. 2011;6:e27131

32. Gowda NM, Wu X, Gowda DC. TLR9 and MyD88 are crucial for the development of protective immunity to malaria. J Immunol. 2012;188:5073-85.

33. Leoratti FMS, Farias L, Alves FP, Suarez-Mútis MC, Coura JR, Kalil J, et al. Variants in the toll-like receptor signaling pathway and clinical outcomes of malaria. J Infect Dis. 2008;198:772-80.

34. Apinjoh TO, Anchang-Kimbi JK, Njua-Yafi C, Mugri RN, Ngwai AN, Rockett KA, et al. Association of cytokine and Toll-like receptor gene polymorphisms with severe malaria in three regions of Cameroon. PLoS One. 2013:8:e81071.

\section{Submit your next manuscript to BioMed Central and we will help you at every step:}

- We accept pre-submission inquiries

- Our selector tool helps you to find the most relevant journal

- We provide round the clock customer support

- Convenient online submission

- Thorough peer review

- Inclusion in PubMed and all major indexing services

- Maximum visibility for your research

Submit your manuscript at www.biomedcentral.com/submit
() Biomed Central 